\title{
ARTÍCULOS
}

\section{EL ESPACIO COMO FILTRO. ACCESO A FONDOS DE MUSEOS ONLINE}

José-Nicolás Del-Río

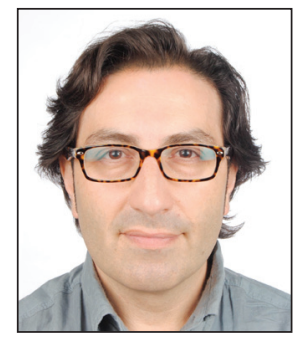

José-Nicolás Del-Río es doctor en arte por la Universidad de Santiago de Compostela, master of arts por la Universidad de Rutgers (EUA) y ha realizado estudios de diseño gráfico en The School of Visual Arts de Nueva York. A lo largo de su carrera ha compaginado su trabajo profesional como diseñador gráfico con la docencia en la Facultad de Ciencias de la Comunicación de la Universidade da Coruña y en masters de la Universidad de Santiago de Compostela. Es investigador asociado al Departamento de Arte de esta universidad. http://orcid.org/0000-0002-4172-0130
Univ. de Santiago de Compostela, Fac. de Geografía e Historia Depto. de Historia del Arte Praza da Universidade, 1. 15782 Santiago de Compostela, España josenicolas.delrio@usc.es

\section{Resumen}

El museo online ha sido definido desde sus inicios como un entorno carente de coordenadas espaciales. A pesar de ello las propuestas de difusión y sensibilización de las webs institucionales siguen habitualmente vinculadas al entorno físico. Esta dependencia produce un desajuste en el acceso a la información online debido a la falta de correlación entre la topología del museo físico y el sistema hipermedia de la Red. Como consecuencia los contenidos online se presentan preferentemente a través de estructuras taxonómicas, típicas de sistemas de catalogación internos, más que estructuras sintagmáticas, propias de la exploración online. Por otra parte el conjunto de referencias al contenedor arquitectónico del museo contribuye a consolidar el espacio físico como patrón del discurso museográfico online y a recuperar el edificio como símbolo identitario del museo, un valor diluido por la virtualidad.

\section{Palabras clave}

Museos online, Webs de museos, Arquitectura de la información, Gestión de datos, Catalogación, Arquitectura, Virtualidad.

\section{Title: Space as a filter. Accessing online museums' content}

\begin{abstract}
The online museum has been defined since its early days as a place devoid of spatial references. However, institutional communication and content mediation in museum websites is commonly linked to the museum's physical space. This connection produces a mismatch due to a lack of correlation between the physical museum's topology and the Net hypermedia system. Consequently, access to museographic online information is mostly laid out in taxonomic structures, typical of gallery cataloguing systems, rather than syntagmatic structures, inherent to online exploration. Furthermore, the profusion of online references to the museum's architectural structure help to consolidate the museum's physical space as a model for the online discourse, as well as to restore the museum's building as an identity symbol, a value blurred by virtuality.
\end{abstract}

\section{Keywords}

Online museums, Information architecture, Data management, Cataloguing, Architecture, Virtuality.

Del-Río, José-Nicolás (2013). "El espacio como filtro. Acceso a fondos de museos online”. El profesional de la información, mayo-junio, v. 22, n. 3, pp. 203-209.

http://dx.doi.org/10.3145/epi.2013.may.02

\section{Introducción}

El museo online es el último eslabón de una cadena de planteamientos conceptuales que lo conciben como entidad no confinada a una estructura arquitectónica. Décadas antes de la aparición de internet, en los años 30, Kiesler imagi- naba un "telemuseo" que transmitiría las obras de las galerías (Storrie, 2006, p. 59). Una década más tarde, Duchamp proponía un museo propio y portátil a partir de su obra Boîte en valisse (1935-1940) y unos años después Malraux (1951) sugería un "museo imaginario" que albergase todas las obras del mundo. En 1960 McLuhan (1964, p. 248) hacía 


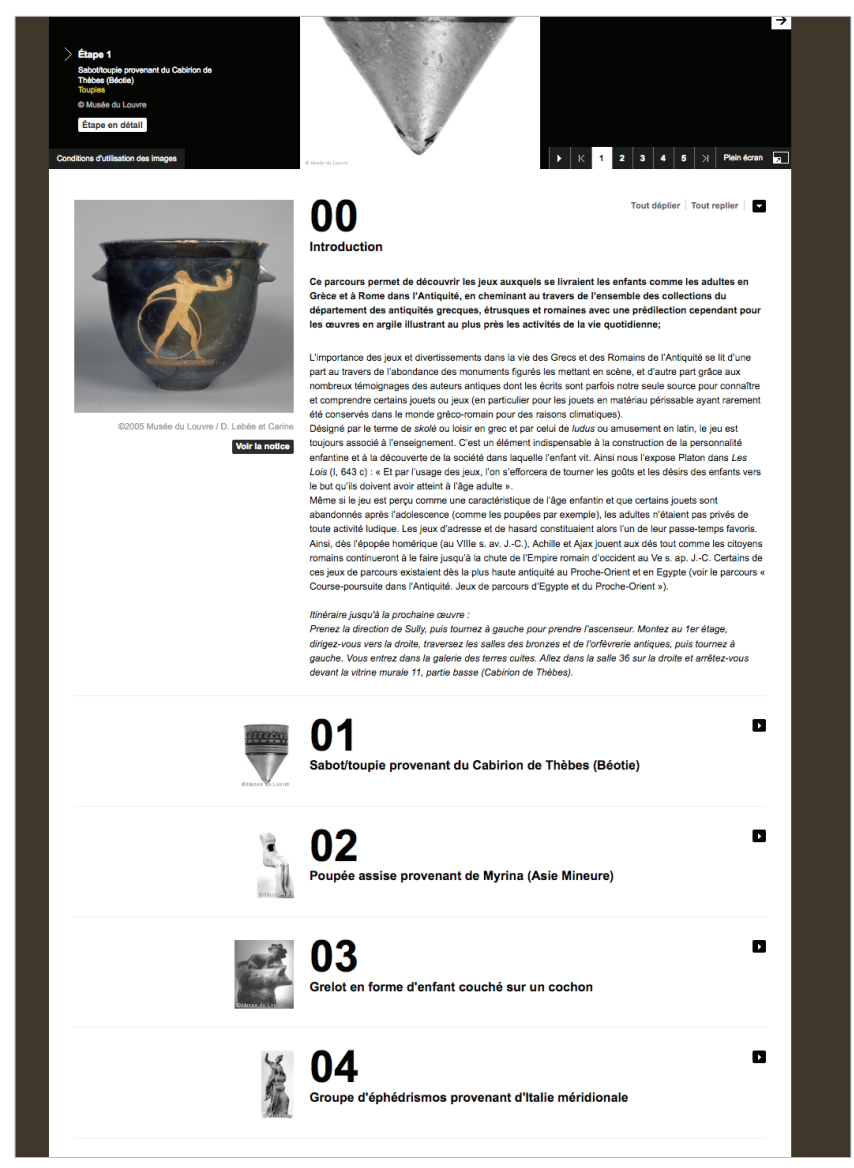

http://www.louvre.fr/routes/jeux-et-jouets

referencia al "museo sin paredes" y más tarde, en 1971, el concepto de "ecomuseo" promovido por Rivière y De Varine, extendía la idea de museo de un edificio a una región (DeCarli, 2004).

\section{El espacio museográfico se convierte en} una pantalla; la distancia deja de ser relevante y la visita adquiere un carácter temporal

La naturaleza digital del museo online, surgido a mediados de los 90, también prescinde de referencias arquitectónicas y convierte la información en su verdadero material de construcción (Kenderdine, 1999). A pesar de ello los contenidos online de difusión y sensibilización de los museos actuales todavía mantienen numerosas referencias al entorno físico. Planos, itinerarios, visitas y exposiciones virtuales, colecciones online o vídeos reproducen modelos y funciones de la experiencia presencial que resultan poco adecuados para la plataforma online. Por una parte las indicaciones cartográficas de posición y orientación que ofrecen los planos no encajan en el espacio online, un entorno creado a partir de datos cuya estructura hipermedia carece de una topología distintiva (Kahn; Lenk; Kasman, 1997, p. 112) y es capaz de adoptar diversos modelos estructurales (Kalbach, 2007, pp. 202-222). Del mismo modo el espacio prediseñado de las salas resulta poco adecuado para mostrar relaciones de significado transversales, superpuestas a las taxonomías expositivas. Las representaciones gráficas online del entorno arquitectónico del museo resultan un referente poco eficaz, incluso superfluo, para los visitantes que acceden al sitio web del museo con una finalidad diferente a la visita presencial. Para ellos el espacio museográfico se reduce a una pantalla en la que la distancia deja de ser una medida relevante (Best, 1993; citada por Ceruli, 1999) y la visita adquiere un carácter temporal (Mitchell, 1996).

Estos desajustes entre el entorno presencial y el espacio virtual constituyen el punto de partida del presente estudio, que analiza cómo la pervivencia de esquemas presenciales en los museos online impone un acceso a la información condicionado por límites espaciales, taxonómicos, secuenciales e incluso semánticos.

Este artículo toma como base de estudio las webs de los 30 museos más visitados del mundo durante 2011 (The art newspaper, 2012). La lista se puede consultar en: http://www.theartnewspaper.com/attfig/attfig11.pdf

Se realizó un análisis entre octubre de 2012 y enero de 2013, que permitió identificar un conjunto de contenidos que incluyen visualizaciones del espacio museográfico y distribuirlos en tres categorías de análisis: visita, fondos y mu$\mathrm{seo}^{2}$. A continuación se muestran los resultados del análisis efectuado, estructurado en estas categorías.

\section{Análisis}

\section{Visita (I). Planos: límites espaciales}

A diferencia de los servicios de acogida presenciales, que abarcan desde propuestas de mediación (visitas comentadas, audio guías, tabletas, etc.) hasta materiales de apoyo (hojas de sala, folletos, códigos QR, etc.), los usuarios virtuales apenas encontrarán contenidos específicamente concebidos para orientarles en su visita online autogestionada. Aunque todas las webs dedican una sección a la visita, habitualmente está enfocada a la experiencia presencial, por lo que el documento más utilizado son los planos $(93,3 \%)$. La mayoría de museos analizados emplea versiones digitales del formato impreso (Tate Modern de Londres, Museum of Modern Art (MoMA) de Nueva York), es decir, un tipo de documento originalmente diseñado para ser consultado en las salas con la finalidad de "establecer una correlación directa entre el espacio físico del museo y la colección que se expone" (Kahn; Lenk; Kasman, 1997, p. 100). Sin embargo este cometido se desvirtúa en la Red, ya que no existe una concordancia entre el espacio real en el que se encuentra el visitante virtual y el museográfico, confinado a un área de píxeles. Desde el punto de vista de los contenidos la consulta de fondos online a través de planos tiene dos consecuencias adicionales:

- limita el acceso a las piezas que se encuentran en exposición e ignora fondos en almacén, préstamo, restauración 0 adquisiciones recientes;

- impone a los visitantes virtuales los mismos condicionantes que un recorrido por las galerías.

http://www.tate.org.uk/file/tate-modern-map http://www.moma.org/visit/plan/atthemuseum/index

Incluso cuando los planos online adoptan un formato interactivo $(33,3 \%)$ y permiten una aproximación de general a particular-de salas a obras- (Louvre de París, Metropolitan 
de Nueva York) la subordinación de los contenidos online a espacios expositivos físicos canaliza conceptualmente la consulta. En opinión de Marshall (1996), el uso de planos online responde realmente a "la subordinación del museo virtual al real, al uso del website como cebo". Desde su punto de vista los planos online sirven "para mantener el control", en otras palabras, para difundir la colección física según esquemas predefinidos, más que para facilitar una exploración abierta de contenidos.

http://www.louvre.fr/plan

http://metmuseum.org/visit/museum-map

No existe una concordancia topográfica entre la disposición de las salas y la diversidad de modelos estructurales hipermedia

\section{Visita (II). Itinerarios, guías y visitas virtuales: límites secuenciales}

Los itinerarios y guías (13,3\% y $10 \%$, respectivamente) son recorridos diseñados en función de conjuntos de piezas con un leitmotiv común. Ambas propuestas superan el contexto y el orden secuencial de las salas para crear una relación supraespacial que propone visitas de punto a punto. La no secuencialidad es una de las características más representativas de la información en soportes digitales interactivos (Fornaciari, 2009, p. 645), de ahí que este tipo de propuestas resulten especialmente adecuadas para el medio online. A pesar de ello los itinerarios y guías que incluyen los museos de este estudio han sido concebidos para la visita presencial. Aunque el Louvre sugiere que los itinerarios pueden imprimirse con anterioridad a la visita o consultarse en la Red, el hecho de estar disponibles online no garantiza que hayan sido diseñados para este medio. De hecho los usuarios se encontrarán con listados de piezas ordenadas numéricamente en función de la proximidad de su ubicación real y de un trayecto cómodo y ordenado por las salas del museo, en vez de múltiples conexiones entre objetos no contiguos. Precisamente la particularidad de los objetos museográficos se encuentra en que sus múltiples capas de significado (autor, obra, fecha, soporte, contexto, interpretación, etc.) dan pie a un amplio número de conexiones que ayudan a "comunicar la significación cultural de las obras artísticas" (Davis; Trant; Vander-Starre, 1996, p. 6). A diferencia del espacio físico donde el visitante descubre nuevas piezas al pasar a una sala contigua, en la Red el descubrimiento que se esconde detrás de cada clic no implica necesariamente un paso más dentro de la misma categoría, sino alguna conexión significativa, lo que fa- vorece "comunicar algo nuevo en comparación con las visitas al museo físico [...], itinerarios personalizados, explorar rutas inusuales, etc." (F-mu.s.eu.m, 2008, p. 54).

http://louvre.fr/parcours

Las visitas virtuales son otro formato de consulta online especialmente popular gracias a Google Art Project- que muestran los fondos del museo en sus lugares de exposición y que resultan particularmente atractivas cuando se trata de edificios históricos o singulares como el Hermitage de San Petersburgo. Ya sea una selección representativa de salas, como la London National Gallery, o un recorrido completo que incluye pasillos y vestíbulos, como el Gyeongju National Museum (Gyeongju, Corea), la visita online se ciñe a los mismos parámetros físicos que itinerarios y guías: recorridos conceptuales marcados por la distribución interna del museo.

http://www.googleartproject.com

http://hermitagemuseum.org/html_En/08/hm88_0.html http://www.nationalgallery.org.uk/visiting/virtualtour http://gvr.museum.go.kr/exh_03.html

\section{Fondos: colecciones y exposiciones: límites taxonó- micos}

En el $80 \%$ de los museos consultados, la ficha técnica de una pieza de la colección está vinculada al espacio físico que la contiene; con idéntico porcentaje las reseñas online sobre exposiciones presenciales también mencionan su localización en el museo. Estas indicaciones de ubicación tienen especial relevancia para las colecciones permanentes ya que en la Red las salas dejan de ser meras referencias para convertirse en una categoría de acceso y catalogación. Por ejemplo la National Portrait Gallery de Londres incluye las

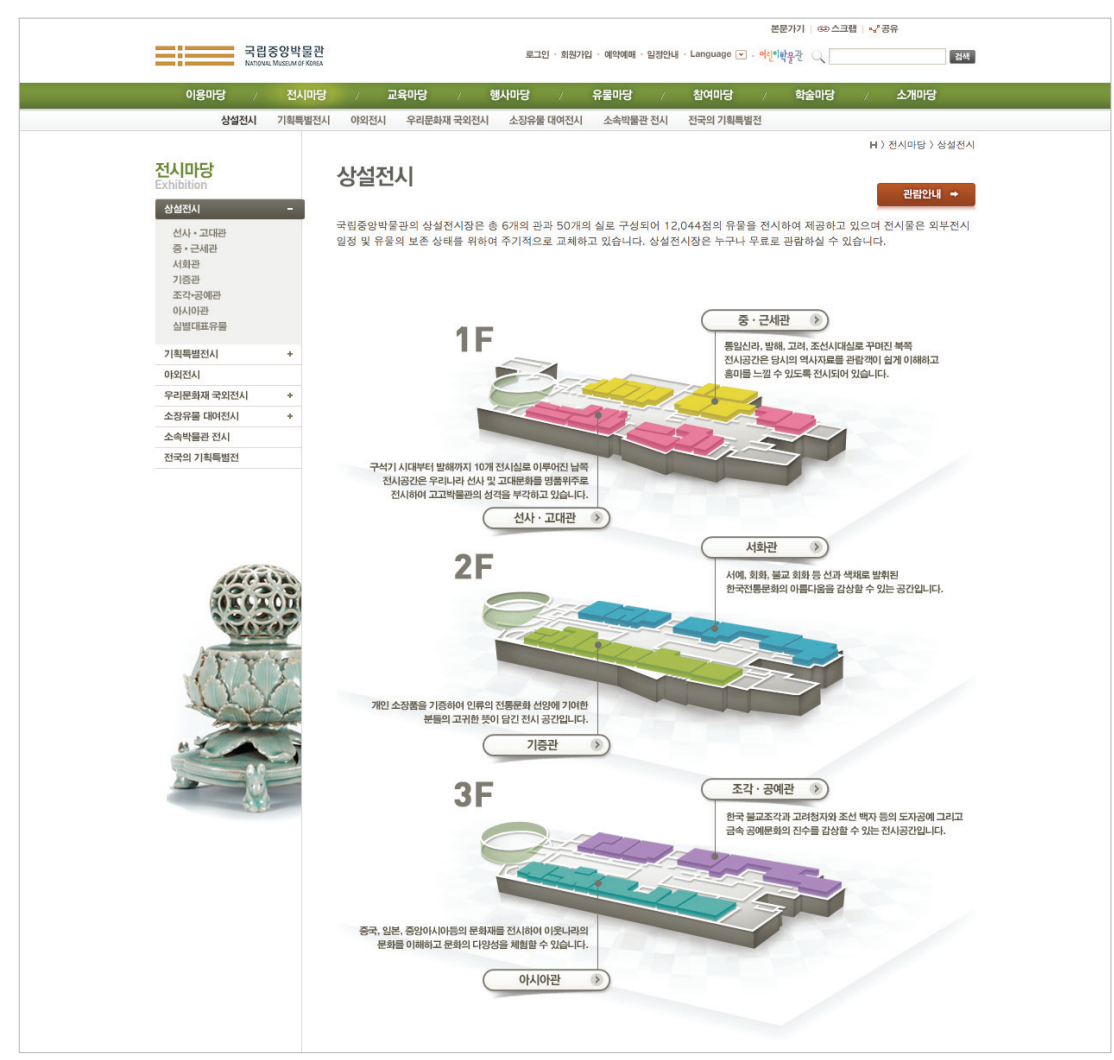

http://www.museum.go.kr/main/publish/view.jsp?menulD=001002001 
salas como uno de los modos de consulta de su colección online, mientras que el Museo Centro de Arte Reina Sofía de Madrid las utiliza como único criterio.

http://www.npg.org.uk/collections/explore.php

http://www.museoreinasofia.es/coleccion/coleccion-1/ presentacion.html

Todas las webs dedican una sección a la visita, pero habitualmente está enfocada a la experiencia presencial

Los sistemas de acceso diseñados en función de la distribución interna del museo no suponen necesariamente un detrimento para la consulta online. Los criterios de la museología física siguen siendo válidos en la Red, especialpresta poca atención y se saca escaso provecho de los modelos propios del medio online. La diferencia fundamental entre los ámbitos museográficos físico y online estriba en dos tipos de presentación de la información; mientras que la gestión y catalogación tradicionales proponen categorías taxonómicas (escultura, pintura española del s. XVII, etc.), la estructura hipermedia posibilita además relaciones transversales (mitología, retrato, etc.) poco frecuentes en la gestión profesional. En otras palabras, la Red favorece una "navegación asociativa" (Wodtke; Govella, 2009, p. 191) más que un afán clasificatorio.

La importancia de valores relacionales entre fondos museográficos online ya había sido señalada por Hoptman (1993, p. 141) que utilizaba el término connectedness (conectividad) para definir este rasgo como una característica "capaz de trascender las categorías tradicionales [museográficas] y de una forma que un libro o una determinada exposición en un museo no pueden hacer". En la misma línea Cameron y Mengler (2009) sugieren la transdisciplinariedad como fórmula para superar "el sistema cerrado de documentación de las colecciones". mente para investigadores y profesionales, no obstante se presentar información contrapuesta o complementaria [...]

\section{El edificio: límites institucionales}

El entorno museográfico físico en el que están expuestas las obras no sólo intensifica la apreciación estética de la visita (MacLeod, 2005, p. 43) sino que también marca pautas que rigen esa experiencia (Mizrach, 2000). En la Red el contenedor arquitectónico ha sido diluido por la virtualidad y, quizá para compensar esta pérdida, el $70 \%$ de los museos analizados dedican a sus edificios secciones individualizadas. En ellas el espacio arquitectónico se pone en valor como una obra merecedora de apreciación estética y se presenta bajo los tres parámetros tradicionales de la cultura museística: obra, catalogación e interpretación. Por ejemplo, el Museo Van Gogh de Amsterdam incluye planos, entorno, arquitectos y publicaciones, y el Centro Pompidou de París un dossier pedagógico.

http://www.vangoghmuseum.nl/vgm/index. jsp?page $=213$ \&lang $=e n$

http://mediation.centrepompidou.fr/education/ressources/ ENS-architecture-Centre-Pompidou/index.html

La pervivencia de esquemas presenciales impone un acceso a la información online condicionado por límites espaciales, taxonómicos, secuenciales e incluso semánticos

Por encima de la valoración artística del edificio y a nivel institucional, la ausencia de un referente arquitectónico en la Red provoca la pérdida de la función simbólica del edificio como indicador de que "en el interior se encuentra algo extraordinario" (Von-Naredi-Rainer; Hilger, 2004, p. 39). Esto se hace aún más patente en las webs de museos en redes sociales. Las páginas y perfiles de estos servicios se caracterizan por un aspecto gráfico prácticamente homogéneo, de ahí que las escasas opciones de personalización sean aprovechadas por algunos museos para situar su sede física como imagen de cabecera. Este es el caso del Prado y del Reina Sofía en Facebook y Twitter, al menos durante el período de esta investigación.

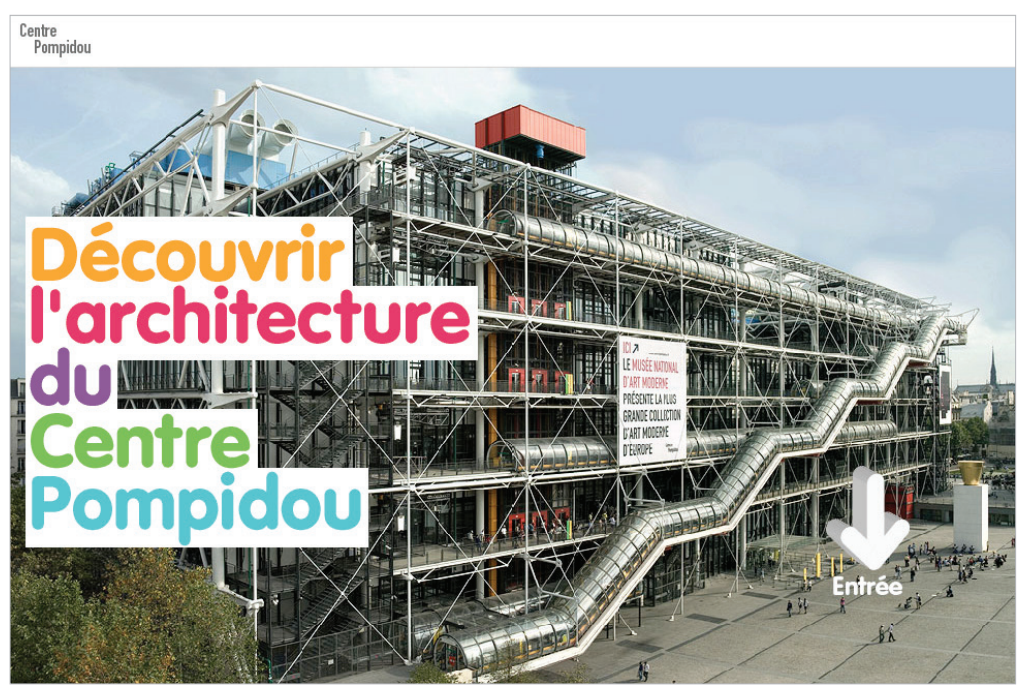

http://mediation.centrepompidou.fr/education/ressources/ENS-architecture-CentrePompidou/index.html ht t ps: / / ww w f facebook.com/ museonacionaldelprado

https://www.facebook.com/museoreinasofia

El espacio institucional también se encuentra presente en los fondos documentales de tipo audiovisual vinculados a piezas de la colección o a exposiciones y eventos temporales. En ellos el entorno museográfico físico en el que se exhiben las obras figura como telón de fondo de charlas, presentaciones, entrevistas..., y funciona a modo de sinécdoque en la que una parte representa el todo. En otros casos, como las visitas guiadas que ofrece el MoMA a través de streaming (emisiones en directo posteriormente archivadas), el recorrido por la salas con un guía que explica las obras dirigiéndose a la cámara intensifica la percepción del entor- 
no museográfico físico. Cualquiera de estas propuestas online remite a los servicios de acogida y mediación habituales de la visita presencial pero, a diferencia de ellos, los visitantes virtuales no encuentran opción de participación. La única posibilidad de interacción se reduce a los controles de reproducción del vídeo:

$h t t p: / / g o o . g l / x G c L F$

\section{Límites semánticos}

Aunque todas las instituciones analizadas disponen de una sección dedicada a la visita, cualquier usuario que consulte sus contenidos (horarios, tarifas, biblioteca...) llegará a la conclusión de que este término es únicamente aplicable a la experiencia presencial. Huelga decir que las visitas al museo físico son esenciales y que es lógico encontrar enfoques promocionales en sus webs, sin embargo la ausencia de recursos específicamente diseñados para facilitar el disfrute de fondos online parece sugerir que los usuarios virtuales podrán consultar, ver, buscar..., pero no "visitar". A juzgar por los casos estudiados, éste es un concepto que los propios museos caracterizan y promocionan en la Red como una actividad claramente presencial. Resulta arriesgado valorar tendencias a partir de un número minoritario de casos pero es posible intuir un patrón similar en las colecciones online.

La Red favorece una navegación asociativa más que un afán clasificatorio

A pesar de que la mayoría de museos siguen identificando claramente sus colecciones como tales, algunas instituciones han sustituido la terminología profesional por con-

ceptos más mundanos como "explorar" (MoMA, British Museum). El cambio no se limita sólo a la nomenclatura ya que los fondos alojados en estas secciones combinan parámetros sintagmáticos y taxonómicos, proponiendo vías complementarias de exploración. También en las exposiciones sería posible intuir una tendencia similar e igualmente minoritaria. Mientras que el término "exposiciones" se reserva para las salas, los proyectos online adoptan nombres específicos en función de su naturaleza. Por ejemplo, Connections (Metropolitan), 3D VR (National Palace Museum, Taipei, Taiwan) o Visites Flash (Quay Branly, París). Este cambio en las denominaciones museográficas apunta a un carácter diferenciador entre la experiencia presencial y online: mientras que el recorrido por las salas mantiene los términos tradicionales de "visita", "colección" o "exposición", una actividad comparable en la Red se identificaría con "exploración" o con proyectos online.

http://www.britishmuseum.org/explore.aspx

http://www.moma.org/explore

http://www.metmuseum.org/connections

http://www.npm.gov.tw/en/Article. aspx?sNo=02000020

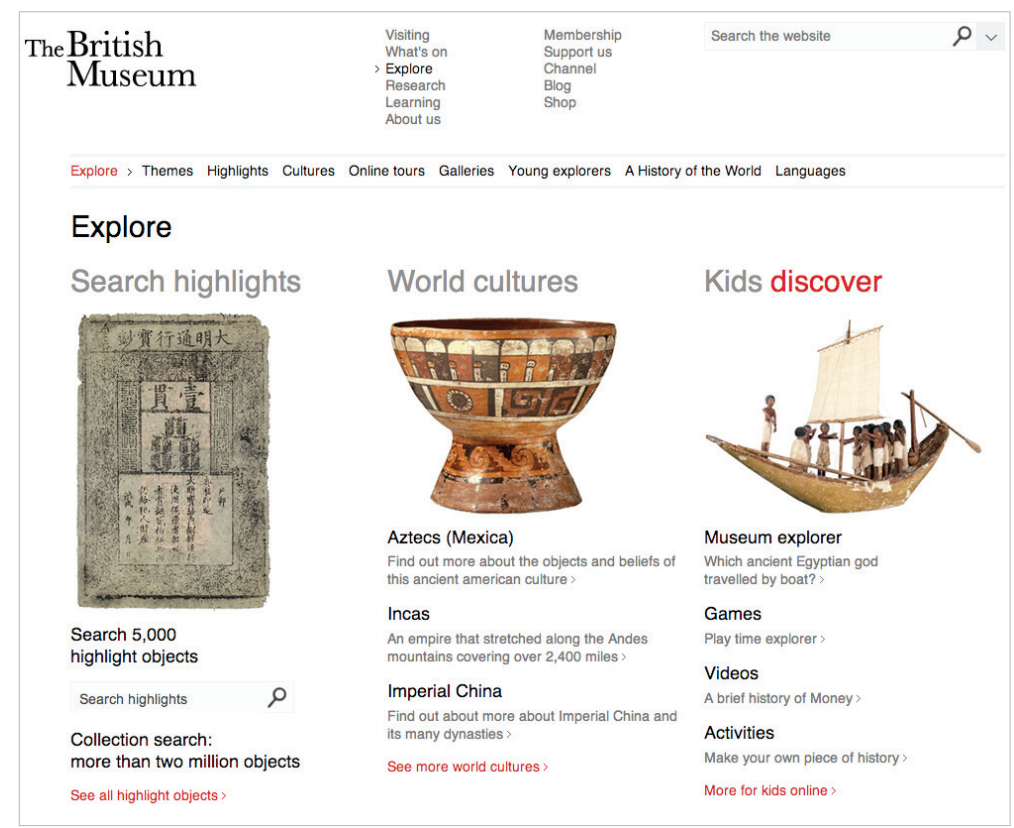

http://www.britishmuseum.org/explore.aspx http://www.quaibranly.fr/fr/collections/visitesflash.html

\section{Pautas de actuación: algunos modelos de referencia}

Todas las propuestas de contenidos online descritas remiten a información presentada en función de parámetros museográficos físicos. No obstante algunas webs analizadas utilizan modelos de consulta relacionales que superan estas limitaciones. Por ejemplo, el Art Institute de Chicago combina en su colección secciones departamentales y nodos temáticos. El Musée d'Orsay de París ofrece enlaces relacionales de una amplia selección de sus fondos permanentes a través del módulo Découverte, y a una escala mucho mayor, el Metropolitan propone su Timeline of Art History, cronología donde se relacionan, fechas, localizaciones y temas junto con índices y búsquedas.

http://www.artic.edu/aic/collections 


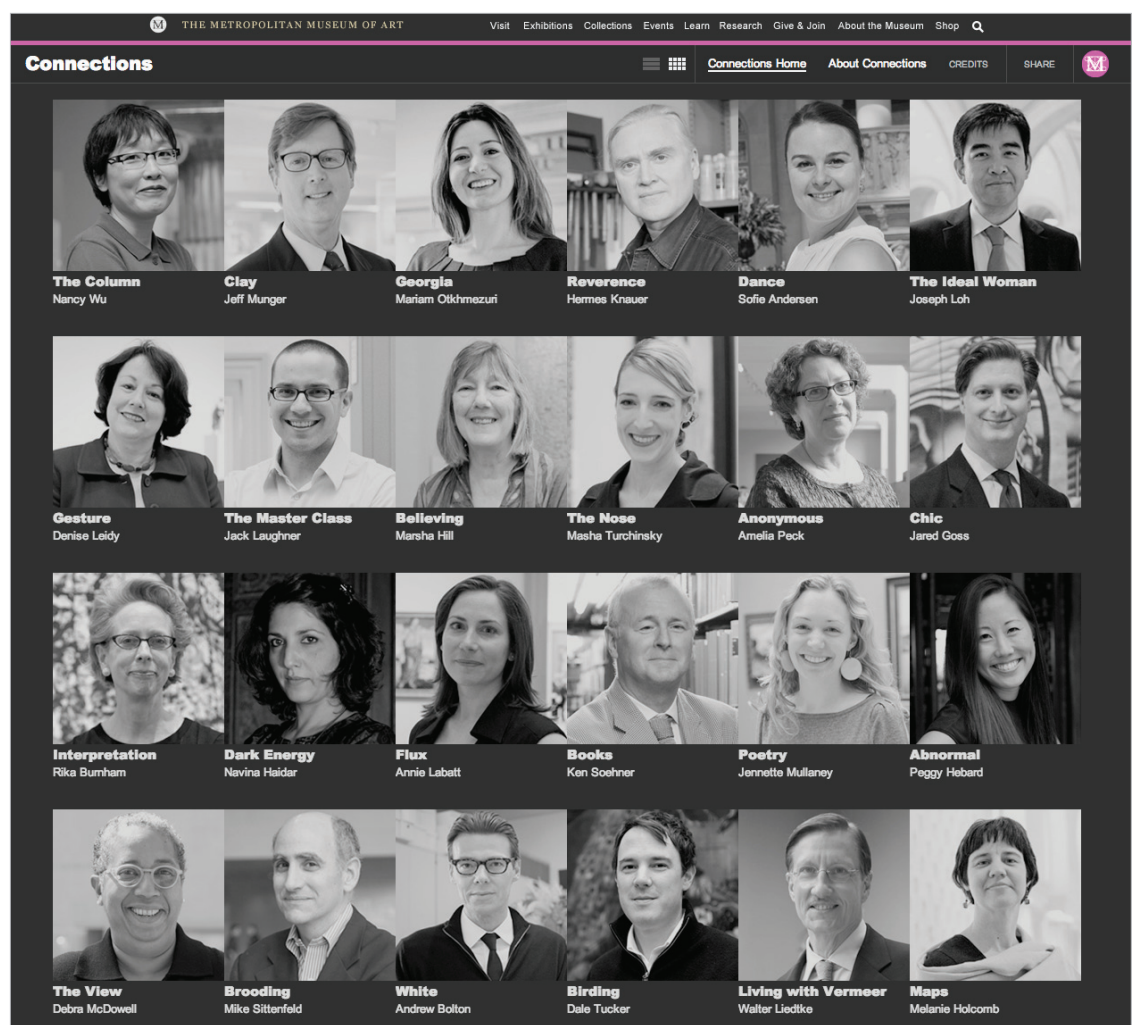

http://www.metmuseum.org/connections

http://www.musee-orsay.fr/fr/collections/decouverte.html http://www.metmuseum.org/toah

El uso de parámetros espaciales fuerza criterios taxonómicos de catalogación, más que patrones sintagmáticos de relación

La catalogación tradicional, resultante de trasladar el espacio físico a la Red, y las colecciones, exposiciones o visitas virtuales producidas a partir de los sistemas de gestión de datos dan lugar a un modelo híbrido de consulta. Sin embargo ambas propuestas conviven de forma paralela, sin existir una integración. Por otra parte en cualquiera de estas iniciativas se echa de menos la contribución de los visitantes para crear una "economía de significado" que comparta la visión institucional y la generada por la comunidad de usuarios (Vestergaard, 2012, pp. 7-9). En esta línea el proyecto steve analizó el uso de etiquetado social en las colecciones de varios museos (Trant, 2009). Este sistema permite a profesionales y visitantes describir obras con sus propias palabras -creando un doble registro lingüístico- y al mismo tiempo establece enlaces transversales a la catalogación profesional de la colección. El resultado es una gestión de contenidos menos rígida y un discurso con múltiples voces.

http://www.steve.museum

\section{Conclusiones}

El estudio de los 30 museos más visitados en 2011 indica que el modelo físico del museo pervive en su versión online tanto visual como conceptualmente. Los fondos online se pliegan mayoritariamente a modelos presenciales que -además de promover implícitamente el espacio físico- imponen autolimitaciones inexistentes en el medio digital: las salas segmentan la información online, el orden secuencial de itinerarios y guías está diseñado por la proximidad física de las piezas, los planos proporcionan un acceso restringido a la colección, las visitas virtuales destacan el contenedor arquitectónico más que las propias obras e incluso algunos términos parecen restringir su significado a la experiencia presencial. El uso de parámetros espaciales impone una difusión y acceso a la información basados en criterios taxonómicos de catalogación, más que en patrones sintagmáticos de relación, consustanciales a la gestión de datos digitales. Las propuestas combinadas de ambos modelos, planteadas por un grupo reducido de los museos analizados, se presentan como una solución equilibrada para cubrir las necesidades de usuarios online diferenciados: expertos familiarizados con las estructuras organizativas del museo, y públicos menos conocedores que utilizan la Red con fines de ocio y aprendizaje informal.

La implantación de contenidos museográficos online destinados específicamente a visitantes virtuales y diseñados a partir de un acceso abierto y flexible a la información permite fórmulas versátiles de consulta -muy difíciles de llevar a cabo en las salas- y además aporta un valor diferenciador a

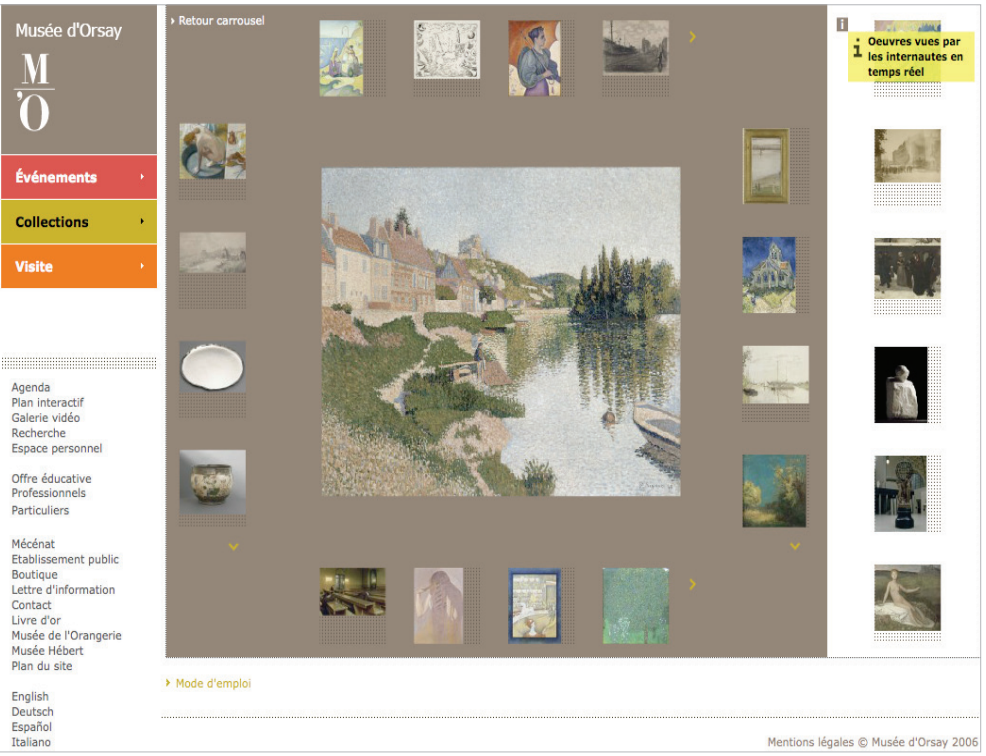

http://www.musee-orsay.fr/fr/collections/decouverte.htm/ 
la experiencia online. No obstante, a juzgar por los museos analizados, ésta es una opción que pocas instituciones parecen dispuestas a fortalecer. De hecho, detrás de la abundancia de modelos presenciales en la Red puede percibirse la intención de consolidar el museo físico como lugar preferente de la experiencia museística.

\section{El museo online consolida el museo físi- co como lugar preferente de la experien- cia museística y lo utiliza en la Red como estructura articuladora de contenidos}

Esto tiene una doble consecuencia: el modelo arquitectónico se mantiene como esquema vertebrador de contenidos online, al tiempo que recupera el edificio como símbolo identitario de la institución. Este es un valor difuminado por la virtualidad que los museos se resisten a perder.

\section{Notas}

1. Ver imágenes en: http://www.moma.org/interactives/exhibitions/1999/ muse/artist_pages/duchamp_boite.html

2. En algunos casos la diversidad de idiomas presentaba una barrera adicional para la consulta. Para evitar posibles omisiones, los museos no disponibles en español, inglés o francés se revisaron a través de Google Translate.

\section{Bibliografía}

Cameron, Fiona; Mengler, Sarah (2009). “Complexity, transdisciplinarity and museum collections documentation: emergent metaphors for a complex world". Journal of material culture, v. 14, pp. 189-218.

http://aaablogs.uoregon.edu/arielles/files/2009/11/ Journal-of-Material-Culture.pdf http://dx.doi.org/10.1177/1359183509103061

Cerulli, Cristina (1999). "Exploiting the potential of 3D navigable virtual exhibition spaces". Papers: Museums and the Web.

http://www.museumsandtheweb.com/mw99/papers/ cerulli/cerulli.html

Davis, Ben; Trant, Jennifer; Van-der-Starre, Jan (1996). Introduction to multimedia in museums: a report by the International Council of Museums Committee on Documentation. La Haya: Icom-Cidoc Multimedia Working Group.

DeCarli, Georgina (2004). Un museo sostenible: museo y comunidad en la preservación activa de su patrimonio. San José de Costa Rica: EUNA. ISBN: 9977652988 http://ilam.org/ILAMDOC/UnMuseo\%20Sostenible.pdf

F-mu.s.eu.m (Form multimedia system for a European museum) (2008). Building a new concept of virtual museum: four case-studies on best practices.

ht tp://ocw2010.ehu.es/mod/resource/view. php?inpopup=false \&id $=1371$
Hoptman, Glen H. (1993). "The virtual museum and related epistemological concerns". En: Barret, Edward. Sociomedia: multimedia, hypermedia, and the social construction of knowledge. Cambridge: MIT Press. pp. 141-160 ISBN 0262521938

Kahn, Paul; Lenk, Krzysztof; Kasman, Magdalena (1997).

"Real space and cyberspace, a comparison of museum maps and electronic publication maps". Museums and interactive multimedia. Archives \& Museum informatics. http://www.archimuse.com/publishing/ichim97/kahn.pdf

Kalbach, James (2007). Designing meb navigation: optimizing the user experience. Sebastopol, CA: O'Reilly Media, Inc. ISBN: 0596528108

Kenderdine, Sarah (1998). "Sailing on the Silicon Sea. The design of a virtual maritime museum". Archives \& museum informatics, v. 12, n. 1, pp. 17-38.

Macleod, Suzanne (2005). Reshaping museum space: architecture, design, exhibitions. New York: Routledge. ISBN: 0415343453

McLuhan, Marshall (1964). Understanding media. London: Sphere Books. ISBN: 8114675357

Malraux, André (1951). "Le musée imaginaire". En: Les voix du silence. Paris: Nouvelle Revue Française. Gallimard. ISBN: 9782071001339

Marshall, Lee (1996). "Code for a Grecian urn”. Wired, 2.09, Sept.

http://yoz.com/wired/2.09/features/museums.html

Mitchell, William J. (1996). City of bits. Space, place and the infobahn. Boston: MIT Press. ISBN: 0262631768

Mizrach, Steve (2000). Lost in cyberspace: a cultural geography of cyberspace.

http://www.fiu.edu/ mizrachs/lost-in-cyberspace.html

Storrie, Calum (2006). The delirious museum. Londres: I. B. Tauris \& Co. Ltd. ISBN: 1845115090

The art newspaper (2012). "Exhibition \& museum attendance figures 2011". The art newspaper, n. 234, pp. 35-43. http://www.theartnewspaper.com/attfig/attfig11.pdf

Trant, Jennifer (2009). Tagging, folksonomy and art museums: results of steve.museum's research.

http://www.museumsandtheweb.com/jtrants/stevemuseum research_report_available

Vestergaard, Vitus (2012). "The hybrid museum: hybrid economies of meaning". En: The transformative museum, 23-25 May, Roskilde, Dinamarca, pp. 1-11.

http://www.dreamconference.dk/wp-content/uploads/2012/03/ vestergaard.pdf

Von-Naredi-Rainer, Paul; Hilger, Oliver (2004). Museum buildings: a design manual. Basilea: Birkhauser Verlag. ISBN: 3764365803

Wodtke, Christina; Govella, Austin (2009). Information architecture: blueprints for the Web. Berkeley, CA: New Riders. ISBN: 0321600800 